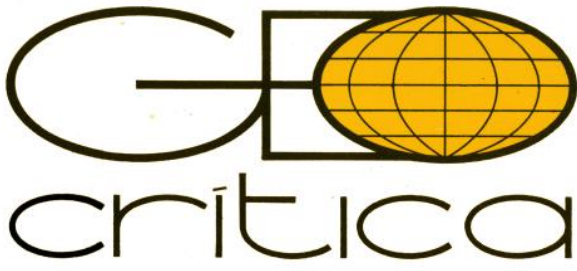

\title{
LAS TECNOLOGÍAS DE LA COMUNICACIÓN E INFORMACIÓN Y SUS IMPLICACIONES EN EL APRENDER/ENSEÑAR GEOGRAFÍA EN LA CONTEMPORANEIDAD: ESTUDIOS DE CASO EN BRASIL
}

\author{
Élida Pasini Tonetto \\ Universidade Federal do Rio Grande do Sul \\ elida.tonetto@ufrgs.br \\ (1Dhttps://orcid.org/0000-000I-7366-8800 \\ Ivaine Maria Tonini \\ Universidade Federal do Rio Grande do Sul \\ ivaine@terra.com.br \\ (1Dhttps://orcid.org/0000-0002-8524-9117
}

\begin{abstract}
Las Tecnologías de la Comunicación e Información y sus implicaciones en el aprender/enseñar Geografía en la contemporaneidad: estudios de caso en Brasil (Resumen)

Viviendo en la Cibercultura, conectados a ella y al mismo tiempo reflejando sus contradicciones, buscamos comprender qué modos de aprendizaje existen en las prácticas comunicativas contemporáneas, a partir de la dimensión espacial de los estudiantes de la enseñanza media en escuelas de Brasil. Entendemos que, en el contexto de la cibercultura y los desafíos por ella generados, son complejos e impactan tanto a los profesores, como al alumnado en sus formas de aprender en el sistema escolar y en sus rutinas personales de estudios. Así, concluimos que las tecnologías digitales de la comunicación e información afectan a los modos como aprendemos y enseñamos Geografía y son también parte de las disputas existentes en la educación formal en general.
\end{abstract} Palabras clave: Geografía; educación; comunicación; cibercultura; escuelas.

Communication and information technologies and their implications in learning $I$ teaching Geography in contemporary times: case studies in Brazil (Abstract)

Living in Cyberculture, connected to it and at the same time reflecting on its contradictions, we seek to understand what ways of learning exist in contemporary communicative practices, based on the spatial dimension of high school students in Brazilian schools. We understand that in the context of cyberculture and the challenges it generates, they are complex and impact both teachers and students in their ways of learning in the school system and in their personal study routines. Thus, we conclude that digital communication and information technologies affect the ways in which we learn and teach Geography and are also part of the existing disputes in formal education in general.

Keywords: Geography; education; communication; cyberculture; schools. 
El contexto de la cibercultura en la contemporaneidad, por medio de la (hiper)conectividad digital, afecta a la sociedad en distintas escalas geográficas; o sea, todos los lugares del mundo, con diferentes intensidades, sufren sus efectos ${ }^{1}$. En la educación formal no es diferente, pues los profesores y estudiantes usan las tecnologías digitales ${ }^{2}$ en sus vidas personales y en las clases, o son incentivados a usarlas, así sienten los desafíos en su cotidiano personal y en el trabajo de la escuela. Pero, más allá del hecho de usar o no usar las tecnologías digitales, la cibercultura y la conectividad digital influyen en el panorama contextual de cómo estudiar, aprender, trabajar y vivir. Así, la cibercultura al mismo tiempo que crea potenciales increíbles para nuestras vidas genera también modos inéditos de explotación humanos, entre otras consecuencias.

Teniendo en cuenta el complejo panorama, adoptamos, en este texto, un sentido "optimista cauteloso" de la cibercultura y del contexto tecnológico actual. ¿Por qué adoptamos la cautela? Porque reconocemos la existencia de problemas, tensiones y disputas sobre las tecnologías digitales en la sociedad, y por supuesto en la educación formal. Sin embargo, aún somos optimistas, porque miramos la dimensión técnica en el cotidiano de la vida, reconociendo las tecnologías integradas en el campo de la cultura y de la educación. Pues un imaginario de homogeneización y de racionalidad instrumental no nos sirve. La época actual nos impone una reflexión compleja del fenómeno técnico.

Adentrando en el tema, entendemos que hay aprendizajes en la comunicación digital en el contexto de la cibercultura. Por eso, a partir de las rutinas de estudios de los alumnos de educación media en Brasil, analizaremos como son los referidos aprendizajes y como la geografía escolar puede ayudar a explicar las prácticas de comunicación en la cibercultura, que son prácticas también "espaciales de comunicación", porque están en contextos materiales de la vida.

La referida comunicación, en general, se realiza de forma espontánea en las aulas escolares, lo que manifiesta una falta de método y organización espacial de la praxis comunicativa. Los resultados empíricos presentados en este texto muestran que los alumnos no tienen criterios para realizar el aprendizaje de la comunicación digital, pero tienen distintos patrones de uso en los lugares materiales (físicos) en que usan las tecnologías digitales para los estudios (clase, corredores, transporte público, casa, etc.).

\section{Entrelazamientos entre cibercultura y aprendizaje}

Entendemos que hay diferentes modos de comprensión del concepto cibercultura y de las fases de las tecnologías de comunicación, incluso mismo las diferentes comprensiones sobre el tema ponen este fenómeno como uno de los más importantes para comprensión de la comunicación en la contemporaneidad ${ }^{3}$.

\footnotetext{
${ }^{1}$ Para Manuel Castells (2003), las Tecnologías de Información y Comunicación (TIC) tienen alcance global, por su capacidad de integrar todos los medios de comunicación y por su interactividad. Ellas están cambiando para siempre nuestra cultura.

${ }^{2}$ Adoptamos el término "tecnologías digitales" para llamar las "Tecnologías de la Información y Comunicación (TICs)", ya que sus bases son en gran parte digitales, incluso es común también el uso del término "Tecnologías Digitales de la Comunicación e Información" (TDCIs).

${ }^{3}$ Autores como André Lemos (2004 y 2010), Lucia Santaella (2007 y 2013) e Pierre Lévy (1993 y 2010) estudian la cibercultura como un tema central entre los fenómenos de la comunicación contemporánea.
} 
Para acercar comunicación y educación, reiteramos la importancia de entender la cibercultura no solamente como un fenómeno técnico, ni tampoco como puramente social, ella es un producto de una fuerte unión entre los procesos tecnológicos y sociales ${ }^{4}$. En el mismo camino entendemos con Pierre Lévy que la cibercultura es un "conjunto de técnicas (materiais e intelectuais), de práticas, de atitudes, de modos de pensamento e de valores que se desenvolvem juntamente com o crescimento do ciberespaço"5.

Para las tecnologías de la comunicación "podemos pensar a história do desenvolvimento tecnológico em três grandes fases: a fase de indiferença (até a Idade Média), a fase do conforto (Modernidade) e a fase da ubiquidade (Pós-Modernidade)", o de la cibercultura, como apunta André Lemos ${ }^{6}$. Siguiendo las ideas del mismo autor, la sociedad de la "cibercultura" es aquella en que las ideologías de la modernidad pierden fuerzas, el tiempo presente gana cada vez más importancia y discursos futuristas pierden adeptos.

Aún en términos de tecnologías de la comunicación, Lucia Santaella ${ }^{7}$ resalta que estamos en la quinta generación de las tecnologías de lenguaje, que son de conexión continúa, representadas por móviles inteligentes, que integran las funciones de otros medios. La cuarta generación fueron las tecnologías de acceso, que son parte de una primera etapa de la "revolución digital", que por medio de un único lenguaje (informático), reúne todos los tipos de texto, voz, imágenes y sonidos, difundidos en diferentes redes, a través de la Internet.

Debemos, también, tener en cuenta que el tema del aprendizaje es amplio, así la principal idea planteada en este estudio es que existen formas de aprender específicas en las prácticas comunicacionales cotidianas de las personas a través de sus dispositivos móviles de conexión continua (móviles inteligentes, tabletas y ordenadores). Las perspectivas sobre aprender que adoptamos en este estudio indican que hay una interacción activa e igual entre los humanos y los objetos ${ }^{8}$.

Siguiendo en la misma línea de pensamiento, planteamos que, para entender las llamadas tecnologías de la cognición, precisamos adoptar una visión no antropocéntrica de la técnica, donde se sostiene el argumento de que tareas y objetos afectan los modos de cognición.

Así consideramos la división de dominios técnicos de la Humanidad en tres grandes momentos, a saber: 1) oralidad; 2) escrita y 3) informática ${ }^{9}$. Este último es el tiempo y el modo en que vivimos con más intensidad; entre tanto, los otros dos siguen existiendo e influyen en cómo pensamos, aprendemos y trabajamos.

Para este estudio enfocaremos las habilidades en el dominio informático, que son las habilidades más evidentes en la contemporaneidad. Estas son: interfaz, que tiene que ver con

\footnotetext{
${ }^{4}$ Como apunta André Lemos (2010).

${ }^{5}$ Pierre Lévy, 2010, p. 17.

6 André Lemos, 2010, p. 52.

${ }^{7}$ Lucia Santaella (2007), apunta 5 fases de las tecnologías de comunicación, en este estudio adoptamos la cuarta y la quinta por estar directamente relacionadas al tema del texto, todavía una generación no sustituye la otra por completo, ellas caminan juntas.

${ }^{8}$ Los autores que se dedican a estudiar ese abordaje son Fernanda Bruno (2002), Pierre Lévy (1993, 2010), Lesley Gourlay y Martin Oliver (2015), Lesley Gourlay (2012, 2014), André Lemos (2010), Vinicius Pereira (2011), Fátima Régis (2011) Fátima Régis, Andreson Ortiz y Raquel Timponi (2012), Martin Oliver (2011, 2012) y Lucia Santaella $(2007,2013)$.

${ }^{9}$ División muy similar adoptada por Pierre Lévy (1993), Vinícius Pereira (2011) y André Lemos (2010).
} 
los proprios dispositivos digitales y que influyen en cómo nos comunicamos; interactividad e hipertextualidad, que están relacionadas con las acciones de las personas y sus dispositivos.

Teniendo en cuenta el dominio informático y sus habilidades es importante analizar atentamente las interacciones entre estudiantes y tecnologías en sus prácticas cotidianas, en sus usos diarios y concretos. En este estudio nos interesa la sociabilidad contemporánea, que es generada justamente por las interacciones sociales y tecnológicas, que están presentes en la vida de los estudiantes, en ellos están también las posibilidades más interesantes de escapar de los discursos hegemónicos o quizá reflexionar sobre ellos.

\section{Consideraciones sobre geografía, educación y comunicación en Brasil}

El estado del arte de las investigaciones de las tecnologías digitales en la enseñanza de Geografía nos lleva a ordenarlas en dos categorías conceptuales, siguiendo también otros estudios realizados: 1) dispositivos en cuanto lenguajes creativos; 2) dispositivos en cuanto lenguajes creadores ${ }^{10}$.

En la primera categoría conceptual el foco reside en la comunicación/transmisión de algún conocimiento geográfico. El lenguaje se presenta como sinónimo de recurso, herramienta o instrumento, generalmente adjetivados de didácticos; como, por ejemplo, recurso didáctico, herramienta didáctica, instrumento didáctico y otros. Esta forma de concebir los lenguajes es oriunda, en especial, de las investigaciones con marco teórico marxista, pero no se limitan a ellas ${ }^{11}$.

En la segunda categoría conceptual, los dispositivos son usados para entender la expresión y la producción del conocimiento en las diferentes obras de la cultura, como los distintos discursos presentes en el cine, en las fotos, en los reportajes y también en los soportes digitales por dónde circulan hoy. Siguiendo la clasificación de Oliveira y Girardi ${ }^{12}$, la gran preocupación de ese grupo es con el conocimiento geográfico producido/construido en cada uno de los lenguajes utilizados en las obras de la cultura.

La búsqueda efectuada en los "Encontros Nacionais de Prática em Ensino de GeografiaENPEG", de 2009 a $2017^{13}$, demostró que una gran parte de los trabajos presentados tienen como estudio principal los dispositivos digitales como lenguajes creativos. Se abordan las tecnologías digitales como un recurso o herramienta para ser utilizado en las clases, apuntan maneras correctas de usarlas, pues entienden que el uso por sí solo es considerado una innovación en las clases.

En todos los ENPEGs se encuentran un número pequeño de trabajos relacionados a la segunda categoría conceptual; o sea, con núcleo de análisis en la producción del conocimiento geográfico en los lenguajes, sea dentro de las escuelas o fuera. Muchos de los trabajos

\footnotetext{
${ }^{10}$ Por Wenceslao de Oliveira Júnior y Giseli Girardi (2011). El término “dispositivo en cuanto lenguaje” hace referencia a todos los dispositivos digitales usados como lenguajes para comunicación en general y no solamente como materiales didácticos.

${ }^{11}$ Wencelao de Oliveira Jr. y Giseli Girardi, 2011.

${ }^{12}$ Wenceslao de Olveira Jr y Giseli Girardi, 2011, p. 01-02.

${ }^{13}$ Ver "Grupo de Trabalho -GT 4-C) O conhecimento da Geografia Escolar, mídias e tecnologias digitais."

Disponible en <http://www.igc.ufmg.br/images/anais_XIIENPEG.pdf〉. [Consulta: 12 de junio de 2019].
} 
encontrados en esa línea tenían críticas sobre las fotos, que en general, son usadas en los materiales didácticos como pruebas de vero similitud sobre la realidad. Este es un tema importante para la enseñanza de Geografía, pues las imágenes tienen cada vez más espacio en los libros de texto ${ }^{14} \mathrm{y}$ en otros materiales didácticos.

Al entender que existen diferentes modos de comprender los "dispositivos digitales como lenguajes", estudiar la innovación y creatividad en las clases escolares, especialmente con las tecnologías digitales es una tarea muy difícil. Por eso, hay que tomar algunas precauciones, teniendo en cuenta los diferentes intereses que se presentan en la introducción de tecnologías digitales en las escuelas, así concordamos con la siguiente idea:

Los centros escolares reciben numerosos estímulos para aceptar las reglas impuestas por los grupos hegemónicos en el sistema de reproducción social. Por ello la incorporación de las nuevas tecnologías representa, como en su momento fue la imprenta y los medios audiovisuales, una oportunidad para difundir el conocimiento entre un mayor número de ciudadanos, pero al mismo tiempo supone un riesgo de una asimilación cultural mecánica, poco crítica y que forme más a clientes que a ciudadanos ${ }^{15}$.

Entonces, como profesores e investigadores de la enseñanza en Ciencias Sociales, es importante estar atentos a las reglas impuestas por los grupos hegemónicos, muchas veces sutiles y disfrazadas, que se interponen en nuestras praxis pedagógicas y en nuestras investigaciones.

\section{Marco teórico y metodológico}

El análisis teórico-metodológico adoptado en este estudio tuvo dos grandes caminos: 1) definición metodológica, que muestra el tipo de estudio y su paradigma; 2) los procedimientos generales, que presentan los criterios de selección de la muestra, los instrumentos de toma de datos, los criterios de organización y los análisis de los datos.

\section{Definición metodológica del estudio}

El diseño metodológico de esta investigación utilizó un enfoque cualitativo, fundamentado en un paradigma de investigación interpretativo, que tiene como método, la investigación en educación, a la luz de las definiciones de estudios realizados ${ }^{16}$. La sustentación del diseño metodológico se basa en la idea que pretende comprender e interpretar a los estudiantes en su medio y en las acciones desarrolladas por cada uno dentro y fuera de la escuela. Así, seguimos una metodología comparativa de estudio de casos con un problema común: el uso de la TIC en el aprendizaje de la geografía.

Así el enfoque cualitativo a partir de los estudios culturales ${ }^{17}$ indican que el posicionamiento del autor/a/investigador/a es la herramienta primordial para la interpretación de lo que ocurre

\footnotetext{
${ }^{14}$ Las investigaciones de Ivaine Tonini $(2002,2011)$ trabajan con ese tipo de abordaje al tratar el tema de las imágenes en libros de texto en Brasil.

15 Jordi Solbes, Xosé Souto, Nelo Traver, 2004; p. 01.

${ }^{16}$ Ver Latorre, 1996 y Bisquerra, 2009.

${ }^{17}$ Denise Gastaldo, 2012, p. 12.
} 
en el campo. Así, tomamos la posición que la inserción en un referencial teórico-metodológico es también política, ética y por fin poética.

\section{Procedimientos generales}

Los procedimientos generales están divididos en tres etapas principales: 1) caracterización de la muestra y aplicación de los cuestionarios; 2) grupo focal en WhatsApp con los estudiantes; 3) observación y registro de espacios escolares y no escolares.

La primera etapa trató de la caracterización de la muestra, o sea la elección del perfil de las instituciones y del alumnado participante. Fueron elegidas para la investigación cuatro instituciones, de tres regiones de Brasil, son ellas: 1) Escuela Estatal de Educación Média Tatakti Kyikateje, de Marabá/Pará/Región Norte, con 10 alumnos participantes; 2) Escuela Estatal de Educación Média Anísio Teixeira, de Marabá/Pará/Región Norte, con 28 alumnos; 3) Proyecto Conexiones de saberes de la Universidade Federal de Alagoas UFAL/Maceió/Alagoas/ Región Nordeste, con 35 alumnos; 4) Instituto Federal do Rio Grande do Sul - IFRS/Osório/ Rio Grande do Sul/Región Sur, con 20 alumnos), como se observa en la figura 1 .

Figura 1. Ubicación de las escuelas participantes

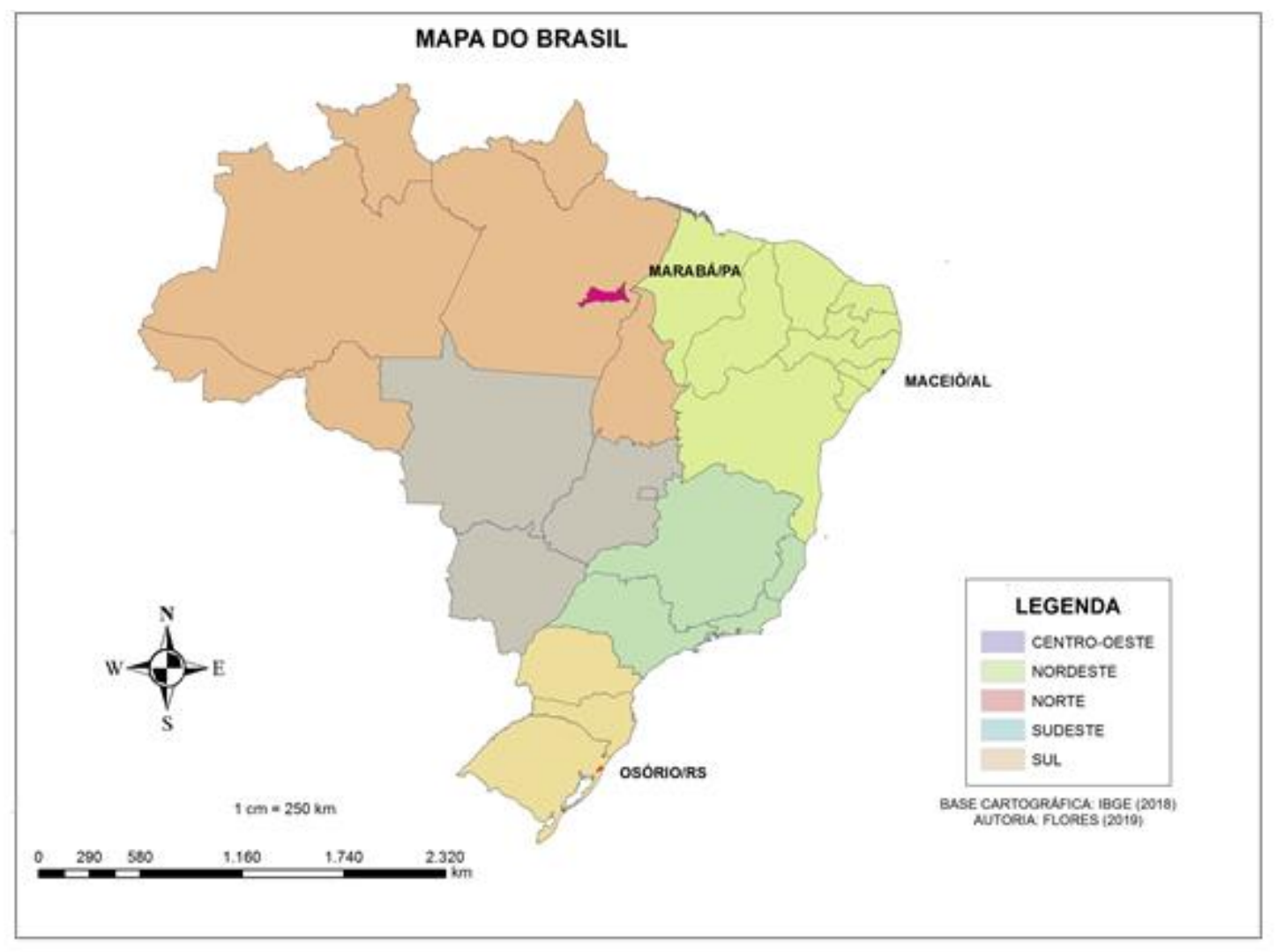


Los criterios de selección de la muestra se basaron en las siguientes características:

a) Los alumnos participantes están en la misma etapa de educación, o sea, en la educación media;

b) Presentan franja de edad semejantes;

c) Están en grupos con compañeros de diferentes localidades y realidades escolares (en el caso del grupo de Osório los alumnos provienen de 8 ciudades, en el proyecto Conexiones de la UFAL los estudiantes vienen de 26 escuelas diferentes);

d) La mayoría de los estudiantes tienen rutinas de estudios paralelos para pruebas externas de ingreso a la universidad, como ENEM (Exame Nacional de Ensino Médio) y/o vestibular;

e) Las escuelas están geográficamente distantes unas de las otras;

f) Las escuelas poseen diferentes contextos sociales e infraestructuras disponibles, sin embargo, todas son públicas.

La validación de los cuestionarios se dio a través de un jurado de expertos, compuesto por cinco profesores, cuatro de ellos trabajan con la formación de profesores y uno con la enseñanza media. La composición tiene dos profesores de la Universidad de Valencia, una profesora de la Universidade Federal do Rio Grande do Sul (UFRGS), una profesora de la Universidade Federal de Alagoas (UFAL) y una profesora del Instituto Federal do Rio Grande do Sul (IFRS/Campus Osório). El agrupamiento de la muestra se basó en dos criterios presentados en el cuadro 1.

\section{Cuadro 1. Criterios de agrupamiento de la muestra}

\begin{tabular}{|c|c|}
\hline Criterio 1 & Criterio 2 + Resultado \\
\hline $\begin{array}{c}\text { Suma de las respuestas de los alumnos en cada } \\
\text { una de las cuestiones. } \\
\text { (Por institución) }\end{array}$ & $\begin{array}{c}\text { Suma de las respuestas de las instituciones de } \\
\text { cada una de las cuestiones. } \\
\text { (Todas las instituciones) }\end{array}$ \\
\hline $\mathrm{X}_{1}=10$ alumnos & $\mathrm{Xt}=\mathrm{X}_{1}+\mathrm{X}_{2}+\mathrm{X}_{3}+\mathrm{X}_{4}$ \\
$\mathrm{X}_{2}=28$ alumnos & 93 alumnos \\
$\mathrm{X}_{3}=35$ alumnos & \\
$\mathrm{X}_{4}=20$ alumnos & \\
\hline Siendo que: & \\
$\mathrm{X}_{1}, \mathrm{X}_{2}, \mathrm{X}_{3}$ e $\mathrm{X}_{4}=$ Respuestas totales (suma de las respuestas de los alumnos por institución) \\
$\mathrm{Xt}=$ Respuestas totales (suma de las respuestas de todas las instituciones)
\end{tabular}

Fuente: Tonetto, 2019.

La etapa segunda fue concretada a través de un grupo focal de WhatsApp, con la participación de un estudiante de cada escuela, con el objetivo de resolver dudas que surgieran en los cuestionarios. Este estudiante actuaba como portavoz de las personas que habían participado en el cuestionario.

Por último, la etapa tres incluye el registro de espacios escolares y no escolares, que tienen una incidencia importante en los modos de comunicarse los estudiantes. Fueron realizadas las 
siguientes actividades: a) visitas presenciales con observaciones y registros fotográficos en espacios culturales, como en el Museo de Paraná (ciudad de Curitiba/Paraná/Brasil), en el Museo de la Fundación Telefónica (ciudad de Madrid/España ${ }^{18}$ ), esa fundación tuvo influencia en el diseño curricular Brasileño -BNCC (2017); b) visitas en espacios escolares y académicos, como en el Instituto Federal do Rio Grande do Sul IFRS/Campus Osório y en la Universidade Federal do Maranhão - UFMA. Las visitas tenían por objeto contrastar las observaciones de las investigadoras con la de los sujetos analizados en las muestras seleccionadas.

\section{Prácticas espaciales de comunicación en la cibercultura}

Los datos de las prácticas comunicativas en espacios físicos fueron recogidos en museos, universidades y escuelas, visitados de modo presencial entre 2015 y 2018 . Al observar dichos espacios verificamos que sus principales características en términos de comunicación generan interacción, colaboración, autoría y movilidad para quién por ellos se mueve. Es una observación de carácter tendencial, en la que se reflejan las rutinas y hábitos que se generan en la comunicación digital entre el alumnado.

En estos espacios tenemos la creación de un espacio intersticial, concepto de Lucia Santaella que indica un tipo de espacio creado entre lo físico y lo digital, especialmente por la inducción de aparatos digitales y también de Qrcodes, que permiten a las personas entrar en conexión directa con informaciones disponibles en la red ${ }^{19}$. Encontramos esta característica en los museos visitados, pero se percibe que es una característica cada vez más presente en diferentes espacios.

Se percibió también que, en los espacios de exposiciones de museos, escuelas y universidades, el visitante/estudiante es llamado a ser "autor", en el momento que elige guías y formatos para el contenido presentado. Verificamos que, se pide del visitante la interacción, colaboración y autoría al visitar las exposiciones y pasillos de las instituciones; o sea, las personas no solo miran, sino que también interactúan con las obras de arte y demás informaciones presentadas.

Todo eso tiene que ver con la gran movilidad generada por dispositivos móviles conectados en red, que piden de las personas un feedback, colaboración y acción. En los dispositivos hay mucha información contextual de los espacios físicos donde se localiza la persona, con la activación del GPS en el celular/móvil; así una búsqueda en un mapa, por ejemplo, será diferente en cada lugar, dependiendo de la posición geográfica de la persona y obviamente de las configuraciones de sus dispositivos.

Además, hay muchas interacciones que ocurren sin presencia de lo digital, pero que son basadas en las funciones de los dispositivos digitales. En ellas se pide interacción con el contenido, como, por ejemplo, murales/paneles, obras de arte físicos e interactivos, en los que las personas son invitadas a exponer sus ideas y completar el contenido, escribiendo con bolígrafos en papeletas etc. En ese sentido percibimos que hay modos de comunicación que dependen de los espacios, pero también hay espacios que exigen de las personas una producción de contenidos, o de sus formatos.

\footnotetext{
${ }^{18}$ Esta colecta de datos ocurrió presencialmente, en el período que una de las autoras estuvo en España (diciembre de 2015 a marzo de 2016), para los estudios presenciales del Doctorado en la Universidad de Valencia.

${ }^{19}$ Lúcia Santaella, 2007, p. 217.
} 


\section{Aprendizajes presentes en las prácticas comunicacionales de la cibercultura}

A partir del material recolectado en el trabajo de campo analizamos algunos de los modos de estudiar de los alumnos de la educación media en Brasil, considerando las dimensiones contextuales en que se incluyen, los espacios de las prácticas de comunicación en la cibercultura y las formas de aprender contemporáneas. Para ello organizamos este apartado en los cuatro temas que estaban en los cuestionarios: 1) Espacios de estudios; 2) Tiempos de estudios; 3) Espacios/Tiempos de estudios; 4) Materiales de estudios.

Encontramos diferentes espacios físicos en que los alumnos se sitúan para estudiar. La tendencia principal se relaciona con lugares con un reglamento normativo menos rígido y con mayor posibilidad de interactuar con otros compañeros: corredor y sala de estudios (sala de clases del propio alumno) son los lugares preferidos para estudiar en la escuela.

Los jóvenes estudian en cuanto circulan por diferentes espacios fuera de la escuela y de la casa. Su primera opción de estudios fuera del hogar fue la casa de amigos y la segunda el transporte público. Esas características, son los trazos del lector ubicuo, descrito por Lucia Santaella como el típico lector de la cibercultura en la era de la movilidad ${ }^{20}$, pues el acceso rápido a las informaciones, a través de dispositivos móviles conectados a internet, permite este hábito de consumo masivo de datos.

Los tiempos en la escuela están bien definidos y regidos por turnos (mañana, tarde o noche), en los que el estudiante necesita estar presente físicamente. Los demás tiempos de estudios son elegidos por los propios estudiantes, porque en ellos hay mayor flexibilidad, el periodo de los fines de semana y festivos son los días preferidos.

El tiempo de concentración de la gran parte de los estudiantes es de 0 a 30 minutos en general, manifestado por 67 de los 93 participantes, siendo el máximo de una hora de estudio. Algo para tener en cuenta sobre el tiempo de concentración personal es que las aplicaciones de mensajes usadas por los alumnos investigados estimulan la falta de atención y acciones multitareas, por medio de los innumerables estímulos que se le ofrecen. Ese tipo de interacción ayuda a reducir cada vez más el tiempo en que el estudiante consigue permanecer en una única actividad. Esta fragmentación de la concentración y la desatención es una característica relevante del aprendizaje en la cibercultura, que dentro de un sistema multitarea es una función necesaria, porque hace que el sujeto consiga alternar de actividad rápidamente, pero en una clase en la escuela puede generar un gran desafío al profesor. En efecto, si dos de cada tres alumnos solo son capaces de concentrarse menos de treinta minutos, y además en un ámbito de multitareas, el tiempo de atención para pensar sobre un problema es escaso.

Al mezclar espacio y tiempo en los análisis se observa una fuerte hibridación entre lo físico y lo digital, pero eso también depende de las condiciones materiales de cada contexto. Así, por ejemplo, en el aula los alumnos no suelen conectarse frecuentemente a sus dispositivos digitales para escuchar música, usar aplicaciones de mensajes y/o de redes sociales. Los principales argumentos para no conectarse en clase están relacionados con la prohibición por la escuela del uso del celular/móvil, y el hecho de que ese tipo de práctica reduce su atención/concentración de la clase. Sin embargo, cuando están en sus casas esas prácticas son realizadas con más

\footnotetext{
${ }^{20}$ Lucia Santaella, 2013, p. 1.
} 
frecuencia. Ello es un argumento muy importante sobre la relevancia de un espacio para generar una actividad, que se habrá de considerar en el momento de la eclosión del teletrabajo.

Otra cuestión expuesta por los estudiantes es que la escuela no está restringida solamente a sus muros; así 77 de los 93 alumnos buscan informaciones sobre el conocimiento escolar y la propia escuela por medio de dispositivos digitales, cuando no están físicamente en ella. Esa búsqueda ocurre por medio de diferentes recursos, como e-mail, chat, páginas web, pero dentro de ellos, se destaca la página de su propia escuela en alguna red social.

Los materiales preferidos para estudiar son, en primera opción el cuaderno y la segunda opción las anotaciones personales en papel. Un total de 65 alumnos afirman fotografiar los propios cuadernos y anotaciones, y 60 de ellos usan el chat para compartirlas; además 86 alumnos buscan materiales alternativos para estudiar, siendo que los sitios de búsqueda mencionados fueron Internet, básicamente Google, WhatsApp y/o YouTube. Entre las demás aplicaciones mencionadas están Geekie Games, Duolingo, Hora do Enem, Descomplica, Menrise; Khan Academy y Stoodi.

De esta manera se han observado que la característica más potente entre todos los modos de estudiar de los estudiantes es que las interacciones se dan con dispositivos, tanto físicos (cuadernos, copias, anotaciones), como digitales (smartphones, computadoras, tabletas). Es decir, hay una lógica de hibridación entre los dispositivos físicos y los digitales, y no una sustitución.

Entre los tipos de aprendizaje destacan modelos personalizados, automatizados y adaptativos, que se encuentran en plataformas de estudios online. En ellas las funciones mecánicas y patrones (programadas) indican respuestas y caminos a los estudiantes. Algunos ejemplos son las plataformas Geekie Games, Duolingo, Menrise, también usados en los Massive Open Online Course - MOOCs. Se supone que el alumnado sigue unas pautas establecidas por la tecnología $\mathrm{y}$, en su origen, por unas directrices empresariales.

Tanto los aprendizajes colaborativos, interactivos, como los ocasionales en red, ubicuos y autoaprendizajes, son formatos que aparecen en prácticamente todos los modos de estudiar de los participantes. Estos aprendizajes se manifiestan en las actitudes de los alumnos en sus estudios, al optar por plataformas online sin indicación de los profesores (autoaprendizaje), al considerar el uso de redes sociales y aplicaciones de mensajes como dispositivos de estudios (en red, ubicuos), al compartir sus materiales (colaborativo, interactivo, distraído), entre otros. Esto nos refuerza la nuestra hipótesis de que el alumnado del siglo XXI desarrolla unas actitudes de aprendizaje escolar al margen de la institución y sus docentes.

Son prácticas de estudios cotidianas y sencillas redefinen la espacialidad del pensamiento, hibridan la mente y alteran tareas cognitivas, principalmente la atención, la percepción y la memoria, ya que los dispositivos digitales y sus interfaces estimulan la falta de atención, exigen percepción veloz y asumen cada vez más las funciones de memorización ${ }^{21}$.

\footnotetext{
${ }^{21}$ Fátima Régis, 2011, p. 10.
} 


\section{Consideraciones finales}

Una primera conclusión obtenida es que hay un aumento considerable de la importancia de los lenguajes, incluyendo el digital y las TICs, en la enseñanza de Geografía, especialmente a partir del año 2002 en Brasil, no solamente en términos de utilización, como también en la investigación. Véanse, por ejemplo: eventos de referencia para la enseñanza de Geografía, como el "Encontro Nacional de Prática de Ensino de Geografia", que agregó a sus discusiones el "Eixo sobre Linguagens no Ensino de Geografía"; o el gran número de grupos de investigaciones oficiales en lenguajes y tecnologías para la enseñanza que figuran en el "Conselho Nacional de Desenvolvimento Científico e Tecnológico - CNPQ". También en el número de tesis con el tema de los lenguajes significativos para la enseñanza de Geografía en Brasil. Se comprueba así la preocupación y la voluntad de abordar esta cuestión.

Como segunda conclusión se percibió que los espacios de las prácticas comunicativas de la cibercultura presentan algunas características fundamentales, como las interacciones que se dan en dos planos complementarios, el físico y el digital, creando un espacio hibrido o intersticial. En ello la persona elige guías y formatos para navegar en los contenidos, interactúa y colabora con su organización, como es el caso de exposiciones de arte en museos, pasillos de escuelas y universidades. Esos espacios cerrados ofrecen cada vez más movilidad, generada por dispositivos móviles conectados en red, que solicitan a las personas feedback, colaboración y acción, siendo muy importante el contexto del espacio físico donde está la persona, producido por la activación del GPS en el celular/móvil. En muchos casos se intenta generar la interacción también sin presencia de lo digital, cuando se pide ayuda de la persona para organizar y agregar contenidos a los espacios, como en los casos de murales interactivos.

Los ejemplos planteados pueden ser incorporados de modo muy productivo a la enseñanza de geografía, llevando al alumnado a entrar en contacto con diferentes miradas sobre el mismo tema, por medio del acceso a diversos materiales (físicos o digitales), tanto en clase como fuera de ella, pero siempre con una presencia activa del profesor/a, para que los análisis sean más reflexivos y los aprendizajes más consistentes. En este sentido, hemos observado la necesidad de profundizar en actividades dialécticas entre los pensamientos espontáneos del alumnado y la generación de una secuencia de actividades de aprendizaje, por parte de los sujetos e instituciones educativas escolares.

Como tercera conclusión entendemos que los aprendizajes presentes en las prácticas comunicativas de la cibercultura están cambiando espacios y tiempos de estudios de los jóvenes y los propios materiales que utilizan. En relación con los espacios es importante considerar tanto los físicos como los digitales, teniendo en cuenta que ambos se mezclan y alteran los patrones de usos e interacciones del alumnado. Los tiempos son cada vez más fragmentados y cortos, la media de tiempo de concentración verificada fue de 0 a 1 hora para los estudios. En ese punto también se percibe una intensa acción multitarea, o sea, el alumno cambia constantemente de tareas mientras estudia. Para el uso de los dispositivos, la habilidad multitarea es importante, como cambiar rápidamente de una ventana a otra en el ordenador o móvil, pero para el estudio de los temas de la Geografía o para las clases en general, puede ser un desafío para los/las profesores/as, visto que muchos de sus contenidos demandan una lectura más atenta y larga, como, por ejemplo, la lectura y reflexión sobre textos, imágenes y gráficos.

Sobre el uso de los materiales, hemos constatado que hay un hibridismo entre lo físico y lo digital, teniendo mucha influencia los espacios físicos sobre la opción del material que son 
utilizados para estudiar. Por ejemplo, en la clase, los estudiantes prefieren no conectarse al móvil, por miedo de perder la atención; en otros espacios al estudiante le gusta conectarse a la red y hacer búsquedas en diferentes fuentes sin la indicación del profesor/a. El espacio del aula escolar genera un comportamiento de estudio que debe ser asumido como condicionante del aprendizaje deseado.

Lo estudiantes siguen usando los resúmenes en papel, pero ahora los comparten en formato digital con sus compañeros de clase. Así hemos visto que no hay una sustitución directa y simple de lo impreso por lo digital, pero si un uso mixto entre los mesmos, siendo esa una cuestión importante para ser pensada en el cotidiano de las clases. Esta característica permite que los maestros puedan hacer nuevos usos del material más tradicional, como, por ejemplo, los libros del texto y mapas en papel, teniendo en cuenta los patrones de interacción de su alumnado y sus posibilidades (acceso o no a la red, uso del móvil, etc.).

Así, planteamos que los aprendizajes en la lógica comunicativa de la cibercultura, aunque, abiertos y complejos, tienden a facilitar la creación de espacios digitales de estudios, como plataformas online de contenidos, en que mediante diferentes dispositivos (móvil, tableta, notebook) y distintas localizaciones físicas (casa, transporte, calle, etc.), en los que el tiempo dedicado a los estudios complementares puede ser ordenado por el estudiante. Pero, mismo con la posibilidad de acceder el contenido lejos de la escuela, hemos identificado que los estudiantes consideran las interacciones presenciales de gran importancia para su aprendizaje, ya que en la escuela hay todo un contexto social y no solamente de acceso a contenidos.

Todo eso se presenta como un desafío para las discusiones sobre tecnología y sociedad, en ese estudio, transpuesto para tecnología y educación, que didácticamente dividimos en tres niveles de relación: táctico, estratégico y retórico ${ }^{22}$. Tomamos como más importante el nivel táctico, este es el nivel de lo cotidiano, el que está en la clase presencial, en la escuela y sus demás espacios. Es en ella en la que están las mejores posibilidades de aprendizaje geográfico, en la que ni lo físico y ni lo digital pueden ser descartados.

Sin embargo, no es prudente dejar de considerar los otros niveles, estratégico (empresas y gobiernos) y retórico (publicidad por los medios) que intentan imponer al profesorado una cantidad de paquetes tecnológicos, muchas veces con versiones de pago, que venden como innovadoras, pero que no consideran a las escuelas y sus contextos. O aún en las políticas públicas de diseño curricular influenciadas por empresas de tecnología educacional, que imponen como calidad e innovación la necesidad de compra de tecnologías digitales, como es el caso de los empresarios que patrocinan la "Base Nacional Común Curricular (BNCC)" en Brasil.

Como cuarta conclusión y, para finalizar, se exponen las preocupaciones para futuras investigaciones en la enseñanza de Geografía y en diferentes áreas de la Educación en general. Así, tanto en las acciones de enseñanza por los/as profesores/as en clase, como en las políticas públicas institucionales, existe el desafío de pensar en los aprendizajes del espacio de la cibercultura y como organizarlos en sus sistemas ya existentes.

Los sujetos involucrados en la educación (principalmente profesores/as) tienen que reflexionar, especialmente, con las apropiaciones por la educación formal de los aprendizajes inherentes a

\footnotetext{
${ }^{22}$ André Lemos, 2010. p. 261.
} 
la cibercultura, muchas de ellas ya incorporadas e inducidas por el mercado. La reflexión sobre tales apropiaciones es quizás más urgente que el propio uso, teniendo en cuenta el avance de las ventas de productos tecnológicos educativos digitales, en formato de "kits didácticos", que muchas veces no consideran las condiciones materiales de las escuelas y de sus sujetos (alumnos y profesores), simplificando la propia concepción de educación como "bien público".

Por último, entendemos que las apropiaciones de los aprendizajes del espacio de la cibercultura están parcialmente abiertas y dependen de discusiones sobre innovaciones en las clases escolares, pero también de decisiones políticas sobre diseños curriculares y del posicionamiento político de las propias investigaciones científicas de las didácticas específicas, especialmente de la Geografía, que urgentemente precisan incluir la dimensión ética sobre la educación como bien público y no como mercancía.

\section{Bibliografía}

BISQUERRA, Rafael. Metodología de la investigación educativa. Madrid, España: La Muralla, 2009. 459 p.

BRUNO, Fernanda. Tecnologias cognitivas e espaços do pensamento. In: FRANÇA, Vera; WEBER, Maria Helena; PAIVA, Raquel; SOVIK, Liv. (Org.). Livro da XI Compós 2002: Estudos de Comunicação. Sulina, 2003, v. 1, p. 193-217.

CASTELLS, Manuel. A galáxia da internet. Rio de Janeiro: Jorge Zahar, 2003. 244 p.

GASTALDO, Denise. Pesquisador/a desconstruído/a e influente? Desafios da articulação teoria-metodologia nos estudos pós-críticos. MEYER, Dagmar; PARAÍSO, Marlucy (Orgs.). Metodologias de pesquisas pós-críticas em educação. Belo Hotizonte: Mazza Edições, 2012, p. 9-13.

GOURLAY, Lesley; LANCLOS, Donna; OLIVER, Martin. Sociomaterial Texts, Spaces and Devices: Questioning 'Digital Dualism'. Library and Study Practices. Higher Education Quarterly <https://onlinelibrary.wiley.com/doi/full/10.1111/hequ.12075>.

GOURLAY, Lesley. Curating, combat or coping? Student entanglements with technologies in HE. 〈http://www.srhe.ac.uk/conference2012/abstracts/0237.pdf>.

GOURLAY, Lesley; OLIVER, Martin. Why it's not all about the learner: a sociomaterial account of students' digital literacy practices. Paper presented at Networked Learning 2014: <http://www.networkedlearningconference.org.uk/abstracts/pdf/gourlay>.

LATORRE, Antonio, DEL RINCÓN, Delio; ARNAL, Justo. Bases metodológicas de la investigación educativa. Barcelona: Hurtado ediciones, 1996. 326 p.

LEMOS, André. Cidade-ciborgue: a cidade na cibercultura. Porto Alegre: Galáxia, 2004. p. 129-148, 10 de junio de $2020<\mathrm{https}$ ///www.researchgate.net/publication/50361527_Cidadeciborgue_a_cidade_na_cibercultura $>$. 
LEMOS, André. Cibercultura: tecnologia e vida social na cultura contemporânea. Porto Alegre: Sulina, 2010. $5^{\mathrm{a}}$ ed.

LÉVY, Pierre. As tecnologias da inteligência: o futuro do pensamento na era da informática. Tradução de Carlos Irineu da Costa. Rio de Janeiro: Ed. 34, 1993. 206 p.

LÉVY, Pierre. Cibercultura. Tradução de Carlos Irineu da Costa. São Paulo: Ed. 34, 2010. 270 p.

OLIVEIRA, Wenceslao; GIRARDI, Gisele. Diferentes linguagens no ensino de geografia. XI Encontro Nacional De Prática De Ensino De Geografia. Goiânia. Anais do XI ENPEG, v. 1. Goiânia, 2011, p. 01-09.

OLIVER, Martin. Technological determinism in educational technology research: some alternative ways of thinking about the relationship between learning and technology, 2011. Journal of Computer Assisted Learning, V. 27(5), p. 373-384.

OLIVER, Martin. Learning with technology as coordinated sociomaterial practice: digital literacies as a site of praxiological study. Proceedings of the 8th International Conference on Networked Learning. 2012. Maastricht. Edited by: Hodgson V, Jones C, de Laat M, McConnell D, Ryberg T \& Sloep P., p. 440-447.

PEREIRA, Vinicius. Estendendo McLuhan: da Aldeia a Teia Global: comunicação, Memória e Tecnologia. Porto Alegre: Sulina, 2011.215 p.

RÉGIS, Fatima. Práticas de comunicação e desenvolvimento cognitivo na Cibercultura. Encontro da Compós. Rio de Janeiro, 2011, p.1-14 <http://compos.com.pucrio.br/media/gt1_f\%C3\%A1tima_regis.pdf $>$.

RÉGIS, Fatima; ORTIZ, Anderson; TIMPONI, Raquel. Tecnologias de Comunicação e cognição. Porto Alegre: Sulina, 2012. 381 p.

SANTAELLA, Lucia. Linguagens líquidas na era da mobilidade. São Paulo: Paulus, 2007. 468 p.

SANTAELlA, Lucia. Desafios da ubiquidade para a educação. São Paulo: Unicamp, 2013. p. 1 <https://www.revistaensinosuperior.gr.unicamp.br/artigos/desafios-da-ubiquidade-para-aeducacao $>$.

SOLBES, Jordi; SOUTO GONZÁLEZ, Xose; TRAVER, Nelo. El impacto de las nuevas tecnologías de la información y de la comunicación en el sistema escolar. Scripta Nova. Revista electrónica de geografía y ciencias sociales. Barcelona: Universidad de Barcelona, 1 de agosto de 2004, Vol. VI, núm. 170 (71) <http://www.ub.edu/geocrit/sn/sn-170-71.htm>.

TONETTO, Élida Pasini. Las tecnologías de la comunicación e información y sus implicaciones para el aprendizajelenseñanza de geografía en la contemporaneidad. (Tesis de doctorado). Universidad de Valencia. Facultad de Magisterio “Àusias March". Programa de Doctorado en Didácticas Específicas. Investigación en Didáctica de las Ciencias Sociales. Valencia: Valencia, 2019. 231 p. 
TONINI, Ivaine. Identidades Capturadas: Gênero, geração e etnia na hierarquia territorial dos livros didáticos de Geografia. (Tese de doutorado). Universidade Federal do Rio Grande do Sul. Instituto de Geociências Programa de Pós-Graduação em Geografia. Porto Alegre: Porto Alegre, 2002. 139 p. <https://lume.ufrgs.br/handle/10183/3191>.

TONINI, Ivaine. Livro didático: textualidades em rede? TONINI, Ivaine et al. (Orgs.) O ensino de geografia e suas composições curriculares. Porto Alegre: UFRGS, 2011, p. 149-159.

(C) Copyright: Élida Pasini Tonetto, Ivaine Maria Tonini, 2020.

(C) Copyright: Ar@cne, 2020.

Ficha bibliográfica:

TONETTO, Élida Pasini; TONINI, Ivaine Maria. Las Tecnologías de la Comunicación e Información y sus implicaciones en el aprender. Ar@cne. Revista Electrónica de Recursos de Internet sobre Geografia y Ciencias Sociales. Barcelona: Universidad de Barcelona, I de septiembre de 2020, vol. XXIV, $n^{\circ} 246$ <DOI: https://doi.org/10.1344/ara2020.246.32210>.

\section{Menú Geo Crítica}

